\title{
OPTICAL COHERENT TOMOGRAPHY IN THE DIAGNOSIS OF INFLAMMATO- RY AND ISCHEMIC OPTICAL NEUROPATHIES (CLINICAL CASES)
}

\author{
Nataliya Moyseyenko
}

Optic neuropathy is a group of diseases of the optic nerve. Using diagnostic techniques such as ophthalmoscopy and perimetry diagnosis are insufficient, as the found abnormalities are not specific for either inflammation or ischemia. Establishing the predominant factor in the pathogenesis of neuropathy is crucial in determining the method of treatment.

The aim of the study: to investigate the features of optic nerve damage using optical coherent tomography (OCT) in optic neuropathy in the acute period.

Materials and methods: two patients with visual impairment were examined.

Result. Patient G. had concomitant rheumatoid arthritis with unregulated Methotrexate therapy. OCT revealed edema of the nerve fibre layer convinced of the predominance of inflammatory neuropathy (atypical neuritis). Pulse therapy with corticosteroids was prescribed, which gave a positive functional effect.

A patient $O$. with pneumonia on the background of Covid-19 with a history of OST showed a decrease in the thickness of the layer of nerve fibres in the lower segment, and in the angio mode-drusen of the optic disc.

Conclusions. Thus, the use of OCT for the diagnosis of optic neuropathies in the acute period showed that under conditions of inflammatory lesions is more characteristic of edema and an increase in the thickness of the layer of nerve fibres. In ischemic neuropathy, on the contrary, a decrease in the thickness of nerve fibres is more characteristic, which correlates in location with the localization of scotoma in the field of view.

Therefore, the use of OCT of the optic nerve will help in understanding the pathogenesis of forms of optic neuropathy. This will help in the choice of treatment tactics

Keywords: optic nerve, neuropathy, OCT, perimetry, scotoma, inflammatory neuropathy, ischemic neuropathy, glaucoma neuropathy, nerve fibres, friends

(C) The Author(s) 2021

This is an open access article under the Creative Commons CC BY license

How to cite:

Moyseyenko, N. (2021). Optical coherent tomography in the diagnosis of inflammatory and ischemic optical neuropathies (clinical cases). ScienceRise: Medical Science, 4 (43), 35-40. doi: http://doi.org/10.15587/2519-4798.2021.237821

\section{Introduction}

Optic neuropathy is a group of diseases of the optic nerve. In the acute form, they are manifested by varying degrees of decreased visual acuity and narrowing of the visual field, disorders of the pupillary afferent reaction. According to the modern classification, among the acute neuropathies the most common are inflammatory $[1,2]$ and ischemic $[3,4]$.

This division is quite conditional, as in the nervous system inflammation and ischemia occur simultaneously, and are accompanied as secondary mechanisms of damage in traumatic [5] and toxic neuropathies [6].

The use of diagnostic techniques such as ophthalmoscopy and perimetry is insufficient, as the found violations are not specific for either inflammation or ischemia. Thus, edema of the disc, which is characteristic of the acute phase, can occur in both neuritis and ischemic neuropathy [7].

In glaucoma neuropathy, an arcuate scotoma occurs more often in the upper half of the visual field, and in ischemic neuropathy in the lower half. Due to which structural elements do such changes occur? It also needs structural clarification [8]. Nevertheless, the establishment of the predominant factor in the pathogenesis of neuropathy in a particular case is crucial for the appointment of a method of treatment.

The aim of the research: to study the features of optic nerve damage using opticocal coherent tomography (OCT) in optic neuropathies in the acute period.

\section{Methods of the research}

During the beginning of 2021, 2 female patients, 44 and 35 years old (4 eyes) with optic neuropathy of various genesis were examined in an outpatient setting at the University Clinic of Ivano-Frankivsk National Medical University (Expert opinion of the Ethics Commission of Ivano-Frankivsk National Medical University, minutes № 111/20 of November 19, 2020). Visometry, ophthalmoscopy and analysis of computer perimeter and OCT data were performed in private institutions of the city.

The work was carried out in accordance with the Code of Ethics of the World Medical Association (Decla- 
ration of Helsinki), all participants gave informed consent to participate in the survey. Ethics Commission of Ivano-Frankivsk National Medical University on compliance with ethical principles, protocol No. 111/19 dated 19.11.2020.

\section{Results}

Clinical case 1. A 44-year-old woman, G., complained of decreased vision in both eyes, which she has been experiencing for about six months. From the anamnesis suffers from rheumatoid arthritis, for a long time took medrol, however, on the advice of a doctor about six months ago, medrol was cancelled and prescribed methotrexate. Vision acuity decreased gradually, unnoticed by the patient. She suffered from iridocy- clitis of her right eye about 1 month ago, and treatment with dexamethasone parabulbar injections gave a temporary improvement, but her vision remained unimproved.

At the time of the examination, in June 2021, the acuity of both eyes with the maximum correction was 0.5-0.6. Relative deficit of afferent reaction of the pupil of the right eye was present.

The fundus showed grayish edema with a faded nasal border of the optic disc of the right eye, sinuosity and full-blooded veins of both eyes.

The results of the perimeter revealed bilateral central scotoma with a mean deviation of sensitivity in the right eye to $-8.68 \mathrm{~dB}$ and the left eye to $-6.68 \mathrm{~dB}$, indicating probable damage to both optic nerves (Fig. 1).

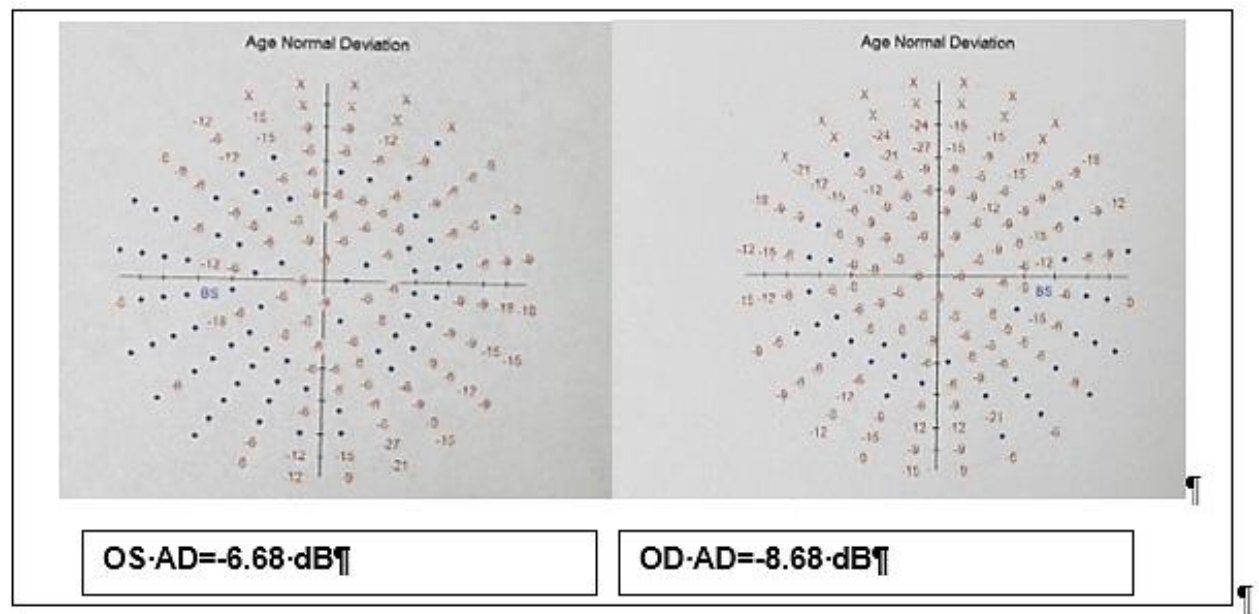

Fig. 1. Computer perimetry of patient G. Perimeter TOMEY: bilateral central scotoma: OS - left eye, OD - right eye, AD mean deviation of sensitivity

OCT of the optic nerves of both eyes were conducted. Edema of the layer of nerve fibers (LNF) of both eyes was detected, which occupied almost all segments in the right eye, and only the lower in the left eye (Fig. 2).

Based on the studies, the diagnosis was made: inflammatory optic neuropathy associated with rheumatoid arthritis [9]. After consultation with a rheumatologist, it was concluded that a similar reaction could occur as a result of the toxic effects of methotrexate [10].

Pulse therapy with corticosteroids was prescribed, visual acuity was restored to 1.0 in both eyes. Observations in the dynamics continue.

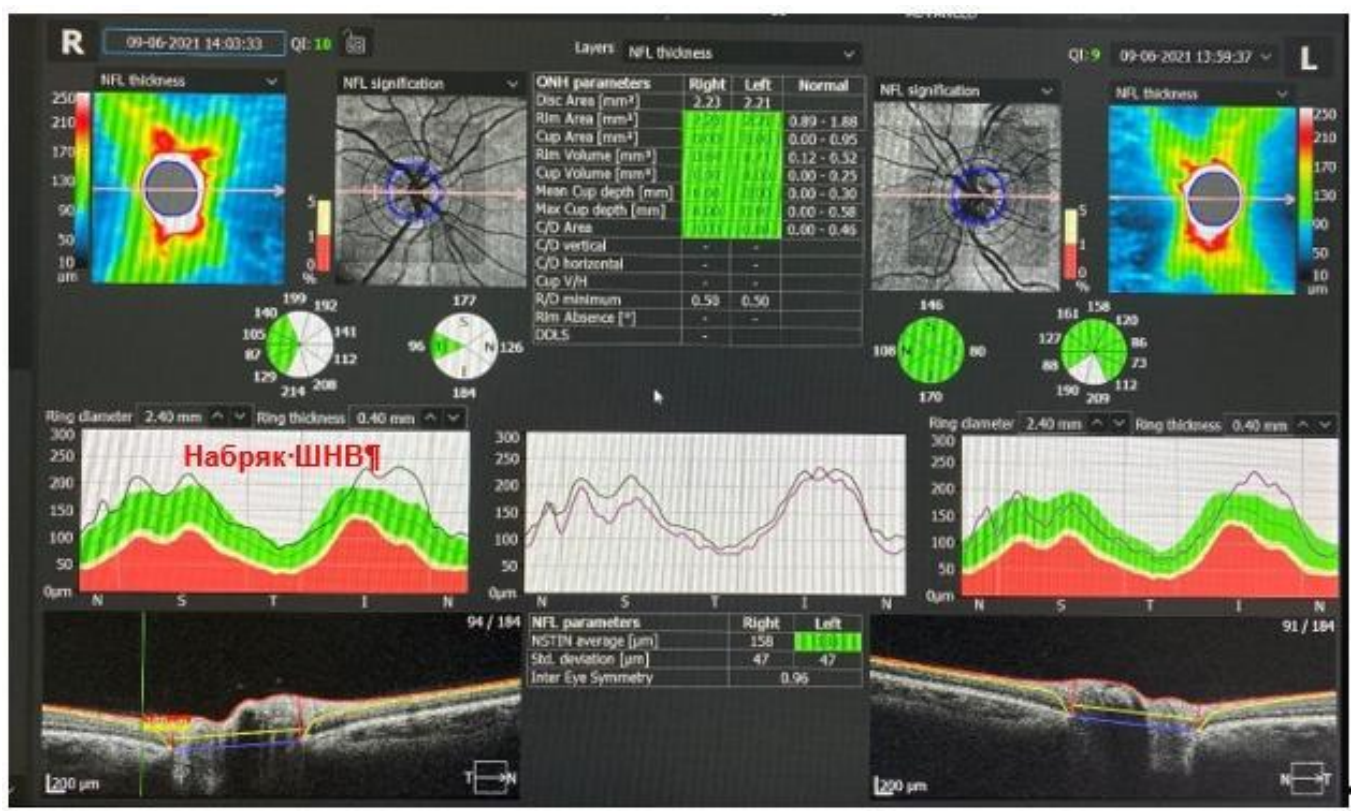

Fig. 2. OCT of the optic nerve of both eyes of patient G .: edema of the layer of nerve fibres (LNF) of both eyes 
Clinical case 2. Patient O., 35 years old. Complaints of decreased contrast, fog is more pronounced on the right, pain behind the eye when moving more on the left, and therefore takes Tobradex for six months. From an anamnesis, patient had Covid-19 in August 2020, blood pressure drops to $130 / 80 \mathrm{~mm}$, which began after recovery. It is at this point that the onset of eye complaints begins.

In the early stages of diagnosis, glaucoma is suspected due to ophthalmic hypertension up to $34 \mathrm{~mm} \mathrm{Hg}$. in both eyes. Perimetry performed for the first time on 10.02.2021 confirmed the hypothesis of glaucoma, as it reveals the expansion of the physiological blind spot, more pronounced in the right eye (Fig. 3).

Repeated perimetry, performed after 20 days, showed bilateral lower central scotoma without the involvement of a blind spot (Fig. 4), which indicates the ischemic nature of optic nerve damage, not glaucomatous [11].

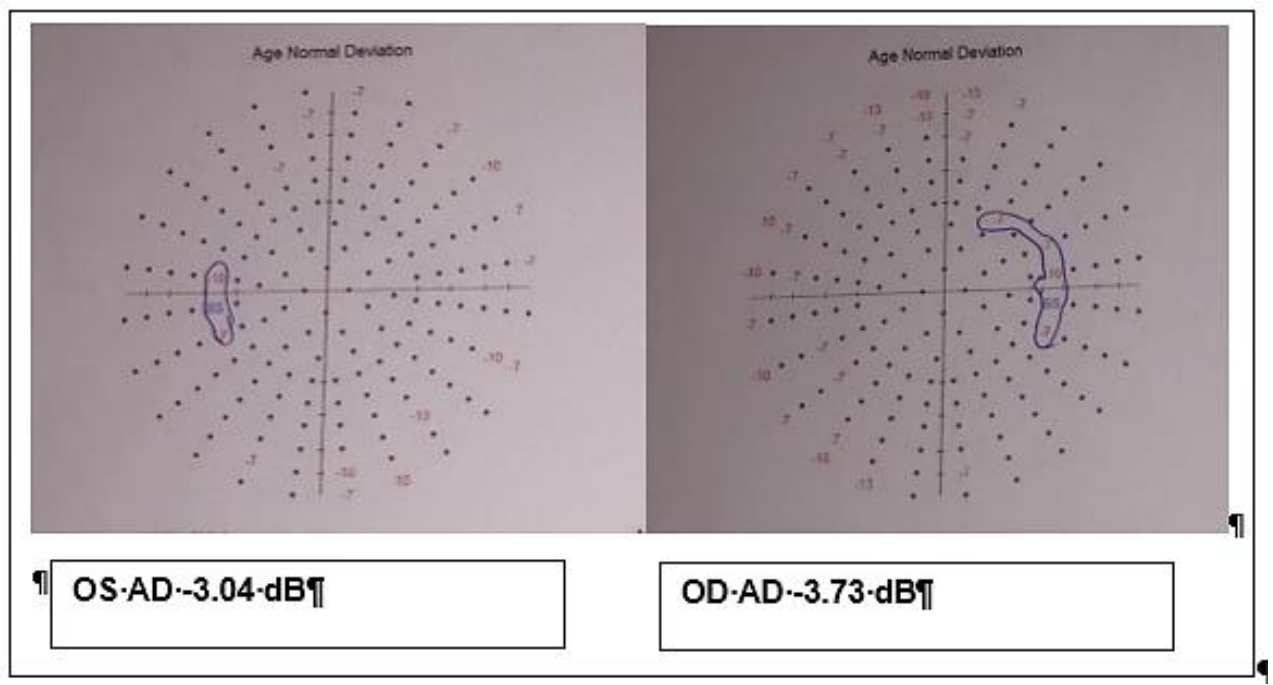

Fig. 3. Computer perimetry of patient O. Perimeter TOMEY. From 10.02.21: enlargement of physiological scotoma, more pronounced in the right eye: $\mathrm{OS}$ - left eye, $\mathrm{OD}$ - right eye, $\mathrm{AD}$ mean deviation of sensitivity

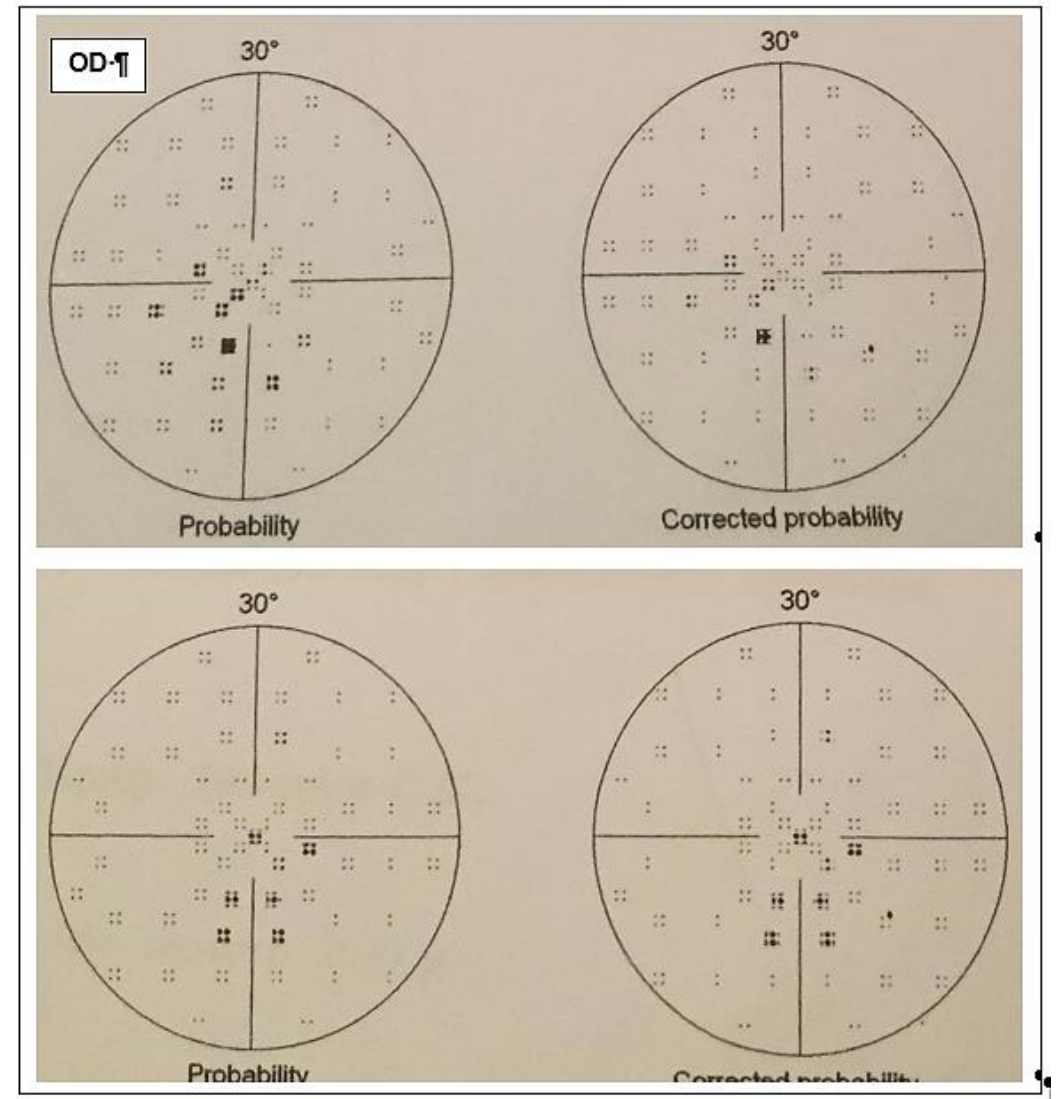

Fig. 4. Perimetry of patient O. from 30.02.21. Octopus 300: bilateral lower-central scotoma 
OCT was performed on June 9, 2021. The lowernasal decrease in the thickness of the nerve fiber layer was revealed (Fig. 5), which is also more characteristic of ischemia [12]. The use of OCT angio regimen allowed to identify drusen on the optic discs of both eyes (Fig. 6), which is a factor in the increased risk of optic nerve ischemia [13]. Aspirin-cardio for the night and luxfen were appointed. Observations in the dynamics continue. In these circumstances, the presence of Covid-19 in clinical history, which promotes hypercoagulation [14], which may be considered an additional factor in ischemia and increased blood pressure, which increase the risk of optic nerve damage in young people in the presence of drusen.

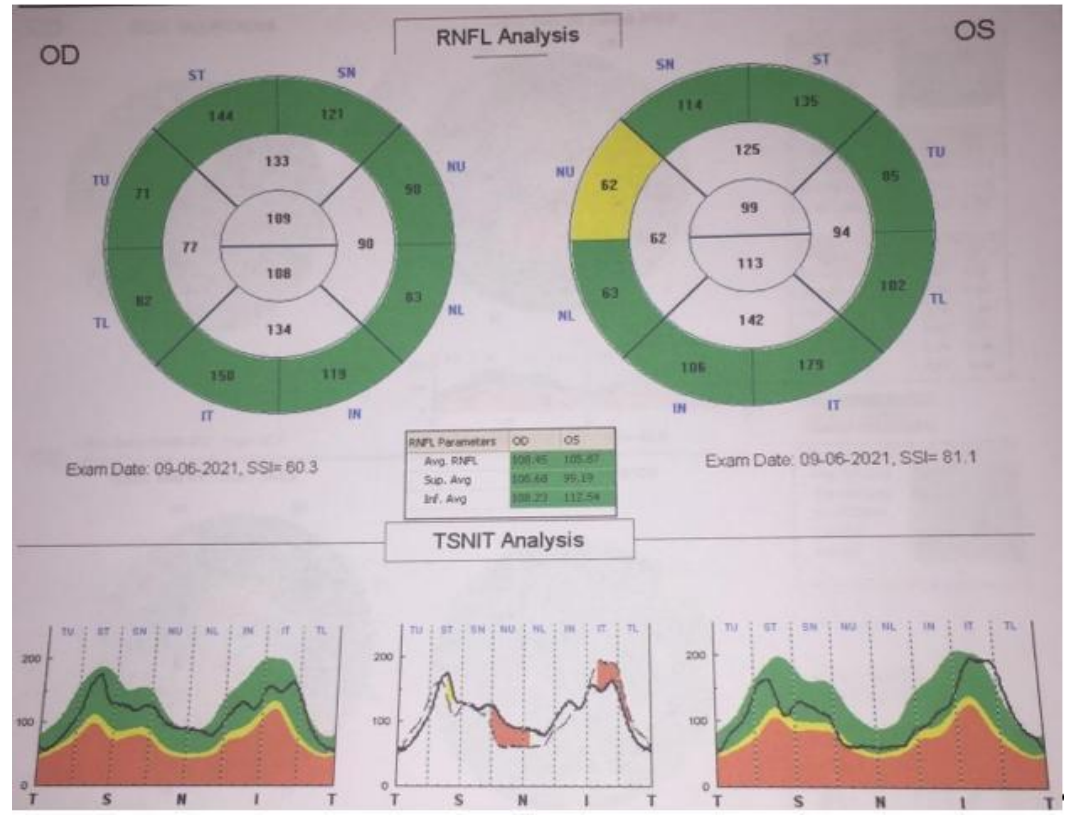

Fig. 5. OCT analysis of LNF thickness of patient O.: decrease in the thickness of the layer of nerve fibres in the lower nasal sector of the left eye
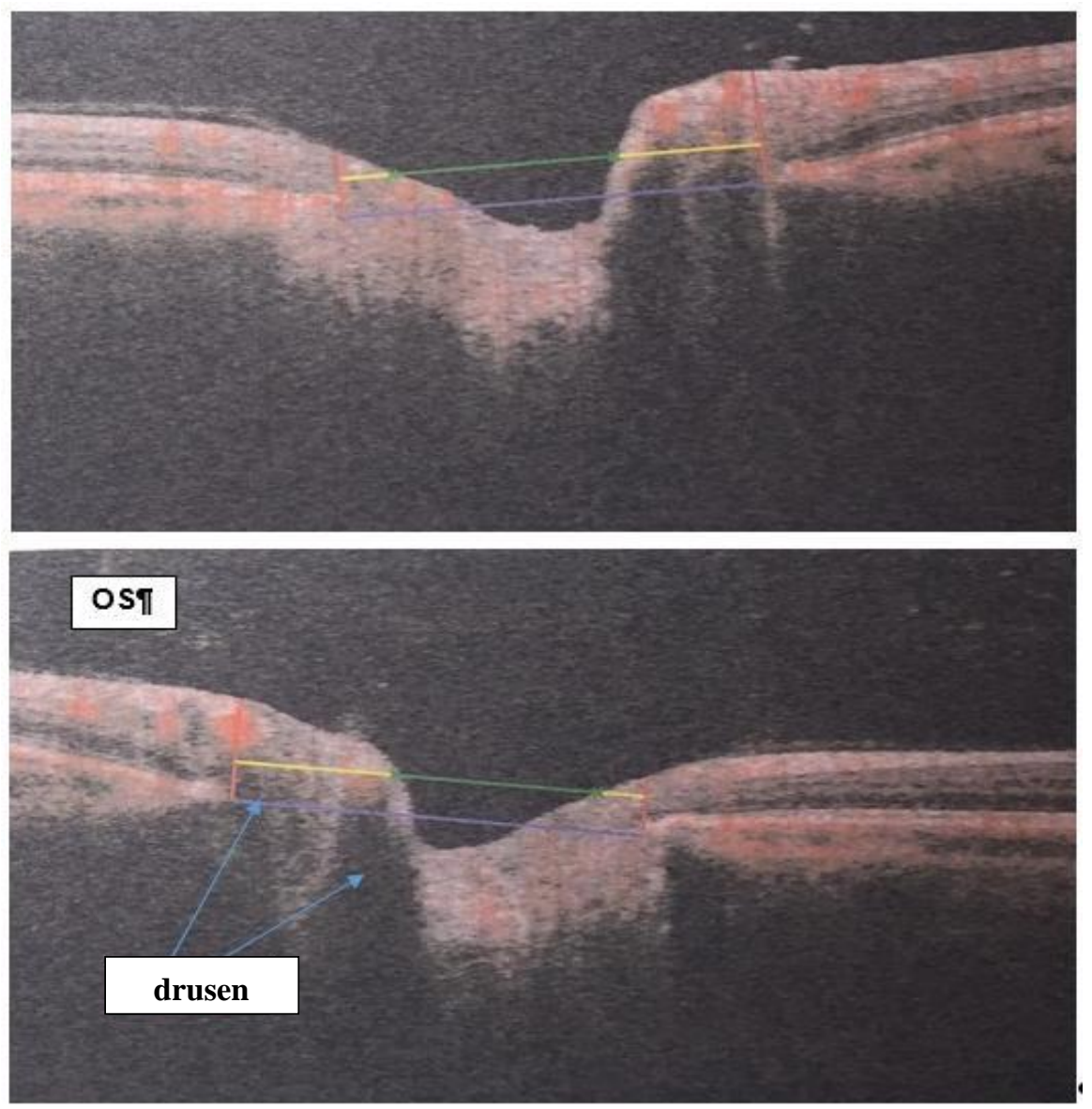

Fig. 6. Angio-OCT of patient O.: drusen of the optic disc 
Regarding increased intraocular pressure, this phenomenon can be explained either by long-term use of Tobradex, or the development of an atypical form of ischemic optic neuropathy [15].

\section{Discussion of research results}

Two patients aged 44 and 35 were examined with approximately the same complaints of decreased vision and fog before the eyes, central scotomas were noted. Examination of the fundus also found no differences.

According to the anamnesis, in both cases there were risk factors that could cause both inflammatory and ischemic lesions of the optic nerve.

In the first patient, concomitant rheumatoid arthritis and unregulated therapy contributed to the development of both atypical neuritis, according to the international classification [9] and, possibly, the toxic effects of methotrexate, as also indicated by previous researchers [10]. To determine further treatment tactics, it was necessary to find out which of the factors played a decisive role.

OCT revealed edema of the nerve fiber layer convinced the predominance of inflammatory neuropathy. Pulse therapy with corticosteroids was prescribed [9], which gave a positive functional effect.

Another patient had a Covid-19 pneumonia and was treated with systemic and topical (due to left eye pain) corticosteroids to treat the inflammatory effects.

Optic nerve damage was initially suspected of glaucoma [11], due to increased intraocular pressure and the formation of arcuate scotoma. However, repeated perimetry showed lower-central scotoma, which is more characteristic for ischemic rather than glaucoma neuropathies.

OST showed a decrease in the thickness of the layer of nerve fibres in the lower segment, which is also characteristic of ischemic lesions of the optic nerve. The use of angio mode of the research allowed to identify friends of the optic disc, which is an additional risk factor for ischemic optic lesions in young people.

According to the recommendations of the North American Association of Neuroophthalmologists, no treatment with proven efficacy has been found in nonarteritic anterior ischemic optic neuropathy [12]. Therefore, aspirin-cardio for the night and luxfen were prescribed.

Study limitations. The difficulty in distinguishing between inflammatory and ischemic changes in the optic nerve is that it is difficult for the nervous tissue to separate these two processes.

Prospects for further research. Study of structural and functional signs of secondary inflammation and ischemia on the background of traumatic or toxic injuries.

\section{Conclusions}

Thus, the use of OCT for the diagnosis of optic neuropathies in the acute period showed that under conditions of inflammatory lesions, probably more characteristic is the swelling and increase in the thickness of the layer of nerve fibres. In ischemic neuropathy, on the contrary, according to our study, there was a decrease in the thickness of nerve fibres. Therefore, the use of OCT of the optic nerve will help in understanding the pathogenesis of forms of optic neuropathy. This will help in the choice of treatment tactics.

\section{Conflicts of interest}

The authors declare that they have no conflicts of interest.

\section{Financing}

The study was performed without financial support.

\section{References}

1. Costello, F. (2014). Inflammatory Optic Neuropathies. CONTINUUM: Lifelong Learning in Neurology, 20, 816-837. doi: http://doi.org/10.1212/01.con.0000453316.60013.52

2. Yassa E.T., Bakbak B. (2019) Chronic Relapsing Inflammatory Optic Neuropathy. The Medical Bulletin of Sisli Hospital, 53 (4), 437-440. doi: http://doi.org/10.14744/semb.2017.58561

3. Wen, Y.-T., Huang, C.-W., Liu, C.-P., Chen, C.-H., Tu, C.-M., Hwang, C.-S. et. al. (2021). Inhibition of Retinal Ganglion Cell Loss By a Novel ROCK Inhibitor (E212) in Ischemic Optic Nerve Injury Via Antioxidative and Anti-Inflammatory Actions. Investigative Opthalmology \& Visual Science, 62 (6), 21. doi: http://doi.org/10.1167/iovs.62.6.21

4. Moyseyenko, N. M. (2019). The pathogenic aspects of traumatic optical neuropathy's neuroprotec-tive therapy. Art of Medicine, 4 (12), 84-91. doi: http://doi.org/10.21802/artm.2019.4.12.84.

5. Sharma, R., Sharma, P. (2011). Toxic optic neuropathy. Indian Journal of Ophthalmology, 59 (2), 137-141. doi: http://doi.org/10.4103/0301-4738.77035

6. Chan, Jane W. (2014) Optic Nerve Disorders Diagnosis and Management. Springer is part of Springer Science: Business Media, 376. doi: http://doi.org/10.1007/978-1-4614-0691-4

7. Grzybowski, A., Barboni, P. (2020). OCT and Imaging in Central Nervous System Diseases. The Eye as a Window to the Brain. Springer International Publishing AG Switzerland is part of Springer Science: Business Media, 343. doi: http://doi.org/10.1007/978-3-030-26269-3

8. Kidd, D. P. (2008). Chapter 7 Inflammatory Optic Neuropathies Not Associated with Multiple Sclerosis. Blue Books of Neurology, 32, 153-190. doi: http://doi.org/10.1016/s1877-184x(09)70037-1

9. Clare, G., Colley, S., Kennett, R., Elston, J. S. (2005). Reversible Optic Neuropathy Associated With Low-Dose Methotrexate Therapy. Journal of Neuro-Ophthalmology, 25 (2), 109-112. doi: http://doi.org/10.1097/01.wno.0000166061.73483.ce

10. Sbeity, Z. H, Baydoun, L., Schmidt S., Loeffler K. U. (2006) Visual field changes in methotrexate therapy. Case report and review of the literature. J. Med. Liban., 54 (3), 164-167.

11. Han, S., Jung, J. J., Kim, U. S. (2015). Differences between Non-arteritic Anterior Ischemic Optic Neuropathy and Open Angle Glaucoma with Altitudinal Visual Field Defect. Korean Journal of Ophthalmology, 29 (6), 418-423. doi: http://doi.org/10.3341/kjo.2015.29.6.418 
12. Fard, M. A., Ghahvehchian, H., Subramanian, P. S. (2020). Optical coherence tomography in ischemic optic neuropathy. Annals of Eye Science, 5, 6-6. doi: http://doi.org/10.21037/aes.2019.12.05

13. Fraser, J. A., Rueløkke, L. L., Malmqvist, L., Hamann, S. (2020). Prevalence of Optic Disc Drusen in Young Patients With Nonarteritic Anterior Ischemic Optic Neuropathy: A 10-Year Retrospective Study. Journal of Neuro-Ophthalmology, 41 (2), 200-205. doi: http://doi.org/10.1097/wno.0000000000000974

14. Sanfilippo, F., La Rosa, V., Oliveri, F., Astuto, M. (2021). COVID-19, Hypercoagulability, and Cautiousness with Convalescent Plasma. American Journal of Respiratory and Critical Care Medicine, 203 (2), 257-258. doi: http://doi.org/10.1164/rccm.202008-3139le

15. Yang, J. M., Park, S. W., Ji, Y. S., Kim, J., Yoo, C., Heo, H. (2017). Postural effects on intraocular pressure and ocular perfusion pressure in patients with non-arteritic anterior ischemic optic neuropathy. BMC Ophthalmology, 17 (1). doi: http://doi.org/10.1186/s12886-017-0441-3

Received date 21.06.2021

Accepted date 23.07.2021

Published date 30.07.2021

Nataliya Moyseyenko, Doctor of Medical Sciences, Associative Professor, Department Ophthalmology, IvanoFrankivsk National Medical University, Halitska str., 2, Ivano-Frankivsk, Ukraine, 76018

E-mail: natalymoyseenko@gmail.com 